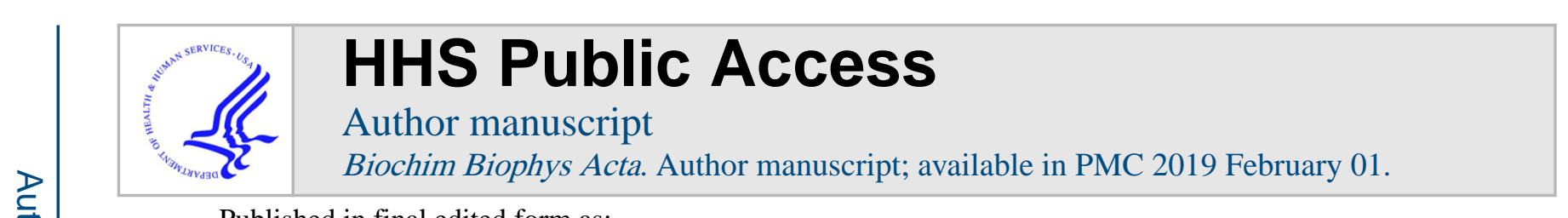

Published in final edited form as:

Biochim Biophys Acta. 2012 July ; 1819(7): 631. doi:10.1016/j.bbagrm.2012.05.002.

\title{
Chromatin in time and space
}

\author{
Gordon L Hager and \\ Center of Excellence in Chromosome Biology, CCR, NCI, USA \\ Lyuba Varticovski \\ Laboratory of Receptor Biology and Gene Expression, CCR, NCI, USA
}

\begin{abstract}
"Space by itself, and time by itself, are doomed to fade away into mere shadows, and only a kind union of the two will preserve an independent reality"
\end{abstract}

Albert Einstein

\begin{abstract}
Understanding chromosome structure, function and dynamics is one of the central issues of modern biology. Chromatin is a specialized structure in eukaryotes which packages, protects and regulates accessibility of nuclear DNA. Vince Allfrey proposed almost fifty years ago (Science 144:559,1964) that chromatin structural modifications, particularly acetylation of histone tails, could also play a significant role in the regulation of transcription. This concept was largely discounted for two decades, until several developments in the late 1980s focused new attention on structural changes in chromatin.
\end{abstract}

Scientists in the NIH intramural program provided a number of key contributions to this evolving view of chromatin and gene regulation. Gary Felsenfeld, with D. Camerini-Otero and B. Sollner-Webb, showed that the H3/H4 tetramer forms the kernel of the nucleosome. Felsenfeld also discovered GATA-1, a protein now seen as important in chromatin interactions. Bob Simpson and colleagues first showed that a nucleosome could occupy selective positions on the DNA template, and also discovered that DNA could be reconstituted onto a cross-linked octamer, proving that the histone subunits were not intertwined with DNA. Gordon Hager and colleagues demonstrated a direct role for nucleosome reorganization in gene regulation by steroid receptors, and later discovered the rapid dynamics of protein movement during these remodeling processes. Carl $\mathrm{Wu}$ isolated the first ATP-dependent chromatin remodeling systems, presaging the characterization of this important family of enzymes.

This rich tradition of chromatin research continues on the NIH campuses. In the National Cancer Institute, the Center of Excellence in Chromosome Biology helps to nurture support for research and postdoctoral training in the chromatin/chromosome field. The CECB center has recently joined forces with the NIH wide chromatin interest group to foster increased communication and interaction with the international community.

The reviews in this issue reflect the breath of activities ongoing within the NIH intramural program. Areas discussed include chromatin domains, chromatin boundaries and insulators, chromosomal proteins, epigenetic modification of chromatin proteins, DNA damage in the 
chromatin context, chromatin dynamics and oscillatory mechanisms, specialized chromosome structures, DNA topology, and other current issues in chromosome biology. We are in a golden age of discovery in the field. The increased understanding of mechanisms involved in chromosome function will continue to provide unexpected opportunities in advancing the cause of improved human health.

\section{Biography}

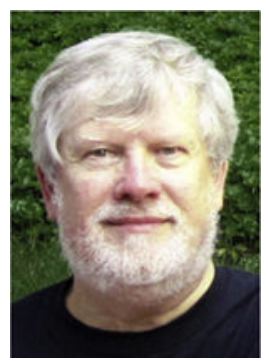

Dr. Hager received his Ph.D. in genetics at the University of Washington, and pursued postdoctoral studies at the Institut de Biologie Moleculaire in Geneva and at the University of California-San Francisco. He is currently Chief of the Laboratory of Receptor Biology and Gene Expression and Chair of the Center of Excellence in Chromosome Biology at the National Cancer Institute, NIH, as well as Adjunct Prof. Of Genetics at George Washington Univ. His program interests include the role of chromatin structure in gene regulation, mechanisms of nuclear receptor function, the dynamics of transcription factor action in living cells, genome-wide organization of regulatory elements, and the architecture of active genes in the mammalian nucleus.

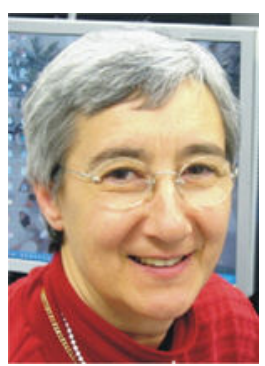

Dr. Varticovski received her MD degree at the U.Valle, Colombia, and pursued basic research training at the U. of Utah and clinical training at the State University of NY Albany School of Medicine. She received specialty training in hematology/oncology at the New England Medical Center, Boston, MA and worked with Lewis Cantley at Tufts Medical School in Boston where she participated in the discovery and characterization of phosphatidylinositol 3-kianse. Her subsequent work involved mechanisms of signaling in cancer cells, drug development and drug resistance in cancer stem cells. She joined NCI in 2003 where she is an Associate Staff Scientist in the Laboratory of Receptor Biology and Gene Expression. Her current work is on normal and cancer stem cells focusing on mechanisms of nuclear receptor, chromatin organization, and transcriptional regulation of gene expression. 